\title{
Sequential Arbitrage Measurements and Interest Rate Envelopes
}

\author{
A. Balbás · S. López
}

\begin{abstract}
This paper proposes new measures that provide us with the level of sequential arbitrage in bond markets. All the measures vanish in an arbitrage-free market and all of them are positive otherwise. Each measure is generated by a dual pair of optimization problems. Primal problems permit us to compute optimal sequential arbitrage strategies, if available. Each dual problem generates a concrete proxy for the term structure of interest rates. The set of proxies allows us to obtain the exact market price of any bond and may measure several effects. For instance, the credit risk spread of nondefault free bonds, or the embedded option price of callable or extendible bonds. The developed theory has been tested empirically.
\end{abstract}

Keywords Portfolio optimization - Sequential arbitrage measurements · Term structure of interest rates $\cdot$ Embedded option premiums

\section{Introduction}

The introduction of general pricing rules is becoming very important in finance. These rules may be justified by theoretical arguments $[1,2]$ or may be implicit in

Research partially supported by Welzia Management SGIIC, RD_Sistemas, Comunidad Autónoma de Madrid (Spain), Grant s-0505/tic/000230, and MEyC (Spain), Grant SEJ2006-15401-C04-03 The authors thank Elizabeth Cabrera (Arizona State University), Alfredo Ibáñez (ITAM-Mexico), Alfonso Novales (Complutense University, Madrid) and the reviewers for helpful comments

A Balbás $(\bowtie)$

University Carlos III of Madrid, Calle Madrid 126, 28903 Getafe, Madrid, Spain

e-mail: alejandro balbas@uc3m es

\section{S López}

Department of Economic Analysis: Quantitative Economics, Autonomous University of Madrid, Calle Tomás y Valiente, 5, 28049 Canto Blanco, Madrid, Spain

e-mail: susana lopez@uam es 
real market prices [3]. Pricing rules are related to the term structure of interest rates (TSIR) in bonds markets. The existence of state prices or pricing rules in a general market, or the existence of TSIR in a bond market, is the necessary and sufficient condition to guarantee that the market is arbitrage free. The arbitrage absence is always assumed in every theoretical approach. However, the empirical evidence seems to reveal that the arbitrage may occur in practice and this fact has motivated several authors to introduce new measures of arbitrage in real markets [4-6]. The present paper modifies the approach of [6] in order to capture those special properties of bond markets and measure their degree of sequential arbitrage. The sequential arbitrage measures are defined by optimal values of dual couples of mathematical programming problems. Primal problems yield the maximum relative (with respect to the price of the purchased or sold bonds) income or profit generated by the implementation of sequential arbitrage. If the measures are larger than zero then they reflect the presence of sequential arbitrage.

Primal problems also provide traders with sequential arbitrage strategies in practice (if available). Actually, primal problems are portfolio choice problems, and it is known that portfolio choice theory is closely related to optimization theory (see [7-9] for recent approaches).

Dual problems generate new measures with a different interpretation. They reflect pricing errors committed by the market. They are never lower than zero and minimize the real price modifications that lead to a sequential arbitrage-free model. Once again, they must vanish to prevent the existence of sequential arbitrage.

Dual solutions also provide proxies (or envelopes) for the TSIR (an exact TSIR cannot exist if the sequential arbitrage occurs). If the market is sequential arbitrage free then the TSIR matches the exact price of all the available bonds. If the market is not sequential arbitrage-free then the set of proxies has more than one element and allows us to compute appropriate spreads for the market price of any bond.

The information contained in option-free bonds is often incomplete and does not generate accurate expressions for the TSIR. So, the negative option price puzzle appears in the literature because callable or extendible bonds are excluded when computing the TSIR in practice, and then negative prices for some embedded options are obtained. We will justify that our measures may be a useful tool to solve this caveat.

The article is organized as follows. Section 2 introduces the basic assumptions. Section 3 presents two dual pairs of optimization problems that lead to complementary measures of sequential arbitrage and envelopes for the TSIR. Section 4 illustrates how the theory enables us to deal with nondefault-free and nonoption-free assets, and Section 5 reports the results of an empirical test implemented in the Spanish market. Section 6 concludes the article.

\section{Preliminaries and Notations}

Consider $n$ arbitrary bonds $B_{j}, j=1,2, \ldots, n$, and denote by $P=\left(p_{1}, p_{2}, \ldots, p_{n}\right)$, $p_{j}>0, j=1,2, \ldots, n$, the row matrix of current prices. Suppose that $\mathcal{T}=$ $\left\{t_{1}, t_{2}, \ldots, t_{m}\right\}$ represents a set of future dates and denote by $a_{i j} \geq 0$ the amount of 
money paid by $B_{j}$ at $t_{i}, i=1,2, \ldots, m, j=1,2, \ldots, n$. In order to avoid some mathematical difficulties, we will impose the following natural inequality whose economic interpretation is obvious:

$$
\sum_{i=1}^{m} a_{i j}>p_{j},
$$

for every $j=1,2, \ldots, n$. Consider finally that $A$ represents the $m \times n$ matrix whose columns are $C_{j}=\left(a_{1, j}, a_{2, j}, \ldots, a_{m, j}\right)^{T}, j=1,2, \ldots, n$, and $\tilde{A}=\left(\begin{array}{c}A_{0} \\ A\end{array}\right)$ represents the $(m+1) \times n$ matrix obtained by adjoining $A$ plus a first row $A_{0}=-P$. If $X=$ $\left(x_{1}, x_{2}, \ldots, x_{n}\right)^{T}$ represents the portfolio composed of $x_{j}$ units of $B_{j}, j=1,2, \ldots, n$, then $P X$ equals the current price of $X, A X$ equals its future payoff and $\tilde{A} X$ equals the whole set of cash flows of $X{ }^{1}$ For $r \in \mathbb{N}$, we consider the following matrices with dimensions $r \times r$ and $r \times(r+1)$ respectively:

$$
I_{r}^{*}=\left(\begin{array}{c}
1,0,0, \ldots, 0 \\
1,1,0, \ldots, 0 \\
\ldots \\
1,1,1, \ldots, 1
\end{array}\right), \quad I_{r}^{* *}=\left(\begin{array}{c}
1,1,0, \ldots, 0 \\
1,1,1, \ldots, 0 \\
\ldots \\
1,1,1, \ldots, 1
\end{array}\right) .
$$

We follow the previous literature in order to introduce the concepts of arbitrage and sequential arbitrage.

Definition 2.1 $X$ is said to be an arbitrage portfolio (AP) if $\tilde{A} X \neq 0$ and $\tilde{A} X \geq 0 . X$ is said to be a sequential arbitrage portfolio (SAP) if $I_{m+1}^{*} \tilde{A} X \neq 0$ and $I_{m+1}^{*} \tilde{A} X \geq 0$.

Arbitrage portfolios have nonnegative cash flows and will pay a positive amount on one date at least. Sequential arbitrage portfolios might imply negative cash flows if they are overcome by the amount of money previously received.

It is known that the (sequential) arbitrage absence may be characterized by the existence of discount factors $\left(\mu_{i}\right)_{i=1}^{m}$ or, equivalently, by the existence of a TSIR. The statement below clarifies this idea.

Theorem 2.1 The model is arbitrage free (respectively, sequential arbitrage free) if and only if there exist $\mu=\left(\mu_{1}, \mu_{2}, \ldots, \mu_{m}\right)$ such that $\mu_{i}>0, i=1,2, \ldots, m$, (respectively, $1>\mu_{1}>\mu_{2}>\cdots>\mu_{m}>0$ ), and $\mu A=P$.

In order to measure the level of sequential arbitrage we will also require some extensions of Definition 2.1.

Definition 2.2 $X$ is said to be a sequential arbitrage portfolio of the second type (STSA) if $P X<0$ and $I_{m}^{* *} \tilde{A} X \geq 0$. $X$ is said to be a strong sequential arbitrage portfolio (SSA) if $P X<0$ and $I_{m}^{*} A X \geq 0$.

\footnotetext{
${ }^{1}$ Given an arbitrary matrix $M$, the transpose of $M$ will be denoted by $M^{T}, M \geq 0$ will mean that $M$ does not contain any negative element, and finally, $M^{+}$and $M^{-}$will denote the classical matrices with non-negative elements such that $M=M^{+}-M^{-}$
} 
Notice that a SAP is a STSA if and only if it is not self-financing, (i.e., the initial price is negative or does not vanish) and a STSA portfolio is a SSA portfolio if and only if the first income (the negative initial price) is not used to overcome future negative cash-flows. Obviously, SSA portfolios are also STSA portfolios, but the converse does not necessarily hold. The absence of STSA and SSA will be characterized in future sections.

\section{Sequential Arbitrage Measurement and TSIR Envelopes}

In order to measure the level of SSA we consider the pair of optimization problems

$$
\begin{aligned}
& \operatorname{Max}-P X, \\
& \text { s.t. } I_{m}^{*} A X \geq 0, \\
& \quad x_{j}+h_{j} \geq 0, \quad j=1,2, \ldots, n, \\
& \quad \sum_{j=1}^{n} h_{j} p_{j} \leq 1, \\
& \quad h_{j} \geq 0, \quad j=1,2, \ldots, n,
\end{aligned}
$$

and

$$
\begin{aligned}
& \operatorname{Max}-P X, \\
& \text { s.t. } I_{m}^{*} A X \geq 0, \\
& \quad x_{j}-k_{j} \leq 0, \quad j=1,2, \ldots, n, \\
& \quad \sum_{j=1}^{n} k_{j} p_{j} \leq 1, \\
& \quad k_{j} \geq 0, \quad j=1,2, \ldots, n,
\end{aligned}
$$

$(X, h) \in \mathbb{R}^{n} \times \mathbb{R}^{n}$ and $(X, k) \in \mathbb{R}^{n} \times \mathbb{R}^{n}$ being the decision variables. Their dual problems are

$\operatorname{Min} \theta$,

s.t. $\mu A+\lambda=P$,

$$
\begin{aligned}
& \lambda_{j} \leq \theta p_{j}, \quad j=1,2, \ldots, n, \\
& \mu_{1} \geq \mu_{2} \geq \cdots \geq \mu_{m} \geq 0, \\
& \lambda_{j} \geq 0, \quad j=1,2, \ldots, n,
\end{aligned}
$$


and

$\operatorname{Min} \theta$,

s.t. $\mu A-\lambda=P$,

$\lambda_{j} \leq \theta p_{j}, \quad j=1,2, \ldots, n$,

$\mu_{1} \geq \mu_{2} \geq \cdots \geq \mu_{m} \geq 0$,

$$
\lambda_{j} \geq 0, \quad j=1,2, \ldots, n,
$$

whose decision variables are $\theta \in \mathbb{R}, \lambda=\left(\lambda_{i}\right)_{i=1}^{n}$ and $\mu=\left(\mu_{i}\right)_{i=1}^{m}$. There is an economic intuition underlying these problems. Indeed, if we focus on problem (2) then $(X, h)$ denotes a pair of portfolios. The first one, $X$, is the strategy to invest, and the objective function leads to the maximization of the sequential arbitrage income $-P X$. First constraint $I_{m}^{*} A X \geq 0$ implies that $X$ is actually SSA if $-P X>0$, and the remaining constraints impose that short sales are bounded from above by a portfolio $h \geq 0$ whose total price cannot be larger than one dollar. The role of portfolio $h$ is clear because the optimal value of (2) is 0 or $+\infty$ if the restrictions involving $h$ are removed. Problem (3) is similar, though portfolio $k$ reflects an upper bound for purchases.

The dual variable $\mu$ of problem (4) may be understood as a proxy for the family of discount factors. Then the first and last constraints in this problem indicate that $\mu$ misprices the available assets, and the second one means that we are minimizing the highest committed error (in percentage) when pricing with $\mu$. Problem (5) is analogous but $\mu$ is overpricing the securities.

Lemma 3.1 Problems (2), (3), (4) and (5) are feasible and bounded. If $£_{*}$ and $£^{*}$ are their optimal values, then $0 \leq £_{*}<1,0 \leq £^{*}$ and $£_{*}=0 \Longleftrightarrow £^{*}=0 \Longleftrightarrow$ the market is strong sequential arbitrage free (SSA-free).

Proof $0 \leq \mathfrak{£}_{*}$ and $0 \leq \mathfrak{£}^{*}$ are clear since $(0,0)$ is feasible for (2) and (3). If $(X, h)$ is (2)-feasible, then $X \geq-h \Rightarrow-P X \leq P h \leq 1$, so $1 \geq \mathfrak{f}_{*}$. Moreover, $1=\mathfrak{f}_{*}$ would lead to the existence of $(X, h)$ feasible and such that $1=-P X \leq P h \leq 1$, and all the terms should be identical. Then,

$$
-P X^{+}+P X^{-}=P h=1
$$

and $P X^{+}=P X-P h \leq 0$ because $X^{-} \leq h$. Since $P X^{+} \geq 0$ is obvious, we have that $P X^{+}=0$ and thus $X^{+}=0$. Expression (6) gives $P X^{-}=P h$, which implies $X^{-}=h$ and $X=X^{+}-X^{-}=-h$. The first constraint in (2) leads to

$$
-h_{1}\left(\sum_{i=1}^{m} a_{i, 1}\right)-\cdots-h_{n}\left(\sum_{i=1}^{m} a_{i, n}\right) \geq 0 .
$$

Expression (1) gives $P h \leq 0$, which contradicts (6). Hence, $1>\mathfrak{f}_{*}$.

In order to prove that (3) is bounded, we only have to prove that (5) is feasible. Expression (1) gives $(1,1, \ldots, 1) A \geq P$, and thus $\lambda=(1,1, \ldots, 1) A-P \geq 0$. Take 
$\theta=\operatorname{Max}\left\{\lambda_{j} / p_{j} ; j=1,2, \ldots, n\right\}$ and we have that $\lambda, \theta$ and $\mu=(1,1, \ldots, 1)$ provide us with a (5)-feasible element.

Finally, if the market is SSA-free then the first constraint in (2) (respectively, (3)) implies that $-P X \leq 0$ whenever $X$ is (2)-feasible (respectively, (3)-feasible) from where $\mathfrak{f}_{*}=0$ (respectively, $\mathfrak{£}^{*}=0$ ). Conversely, suppose that $£_{*}=0$. Then, if $I_{m}^{*} A X \geq 0$, take

$$
Y= \begin{cases}X /\left(P X^{-}\right), & \text {if } P X^{-}>1, \\ X, & \text { otherwise }\end{cases}
$$

and

$$
h= \begin{cases}X^{-} /\left(P X^{-}\right), & \text {if } P X^{-}>1, \\ X^{-}, & \text {otherwise. }\end{cases}
$$

$(Y, h)$ is clearly (2)-feasible. Hence, $P Y \geq 0$ and thus $P X \geq 0$. Consequently, there are no portfolios satisfying Definition 2.2 . If $£^{*}=0$, we can similarly proceed, with straightforward modifications.

Theorem 3.1 There are no SSA portfolios if and only if there exists $\mu_{*}$ such that $P=\mu_{*} A$ and $\mu_{* 1} \geq \mu_{* 2} \geq \cdots \geq \mu_{* m} \geq 0$.

Proof According to the previous lemma, the absence of SSA holds if and only if the optimal value of (4) vanishes.

Lemma 3.2 Suppose that $£_{*}>0$. If $\left(X_{*}, h_{*}\right)$ solves (2) and $\left(X^{*}, k^{*}\right)$ solves $(3)$, then $X_{*}^{-}=h_{*}, P h_{*}=1, X^{*+}=k^{*}$ and $P k^{*}=1$.

Proof Let us prove that $X_{*}^{-}=h_{*}$. Since $X_{*}^{-} \leq h_{*}$ and the components of $P$ are strictly positive, it is sufficient to see that $P X_{*}^{-}=P h_{*}$. Since $P h_{*} \leq 1$, it is sufficient to see that $P X_{*}^{-}=1$. Suppose that $P X_{*}^{-}<1$. Obviously, $P X_{*}^{-}>0$ because $P X_{*}^{-}=0$ would imply

$$
£_{*}=-P X_{*}=-P X_{*}^{+}+P X_{*}^{-}=P X_{*}^{+} \leq 0 .
$$

Set $Y=X_{*} /\left(P X_{*}^{-}\right)$and $h=Y^{-}$and we have that $(Y, h)$ is feasible and such that

$$
-P Y=\left(-P X_{*}\right) /\left(P X_{*}^{-}\right)=\mathfrak{£}_{*} /\left(P X_{*}^{-}\right)>\mathfrak{£}_{*}
$$

and we have a contradiction. The remaining results can be proved with similar arguments.

Theorem 3.2 Suppose that $£_{*}>0$. The following statements are true:

(a) $£^{*}=\frac{£_{*}}{1-£_{*}}, £_{*}=\frac{£^{*}}{1+£^{*}}$ and $£_{*} \leq £^{*}$.

(b) Assume that $\left(X_{*}, h_{*}\right)$ solves (2) and $\left(X^{*}, k^{*}\right)$ solves (3). Then, $X^{*}=k^{*}-(1+$ $\left.£^{*}\right) h_{*}, X_{*}=\left(1-£_{*}\right) k^{*}-h_{*}$ and $X^{*}=\left(1+£^{*}\right) X_{*}$. 
Proof Inequality $\mathfrak{f}_{*}>0$ implies that $\mathcal{A}=\left\{X \in \mathbb{R}^{n} ; P X<0, I_{m}^{*} A X \geq 0\right\}$ is nonvoid. Consider the functions $\phi_{i}: \mathcal{A} \longmapsto \mathbb{R}, i=1,2$, given by

$$
\begin{aligned}
& \phi_{1}(X)=\frac{-P X}{P X^{-}}, \\
& \phi_{2}(X)=\frac{-P X}{P X^{+}} .
\end{aligned}
$$

Notice that denominators do not vanish in the definition above. Indeed, $P X^{-}=0$ would imply $P X=P X^{+} \geq 0$, contradicting $X \in \mathcal{A}$, and $P X^{+}=0$ would imply $0>P X=P X^{-}$, which makes

$$
(Y, h)=\left(X /\left(P X^{-}\right), X^{-} /\left(P X^{-}\right)\right)
$$

(2)-feasible and therefore $£_{*}=1$, contradicting Lemma 3.1.

Expressions $0<\phi_{1}(X)<1$ and

$$
\phi_{2}(X)=\frac{\phi_{1}(X)}{1-\phi_{1}(X)}
$$

are obvious. Since $[0,1) \ni t \longrightarrow t /(1-t) \in[0, \infty)$ is a one-to-one increasing function, the problems

$$
\operatorname{Max}\left\{\phi_{i}(X) ; X \in \mathcal{A}\right\}
$$

$i=1,2$, attain the optimal value at the same solutions. As said above, if $X \in \mathcal{A}$, then $(Y, h)=\left(X / P X^{-}, X^{-} / P X^{-}\right)$is (2)-feasible, and therefore $(-P X) /\left(P X^{-}\right) \leq £_{*}$. Furthermore, the previous lemma implies that $X_{*}^{-}=h_{*}$ and $P h_{*}=1$, from where

$$
\phi_{1}\left(X_{*}\right)=\left(-P X_{*}\right) /\left(P X_{*}^{-}\right)=-P X_{*}=£_{*}
$$

and $X_{*}$ solves (8). Similarly, $X^{*}$ solves (8) and $\phi_{2}\left(X^{*}\right)=\mathfrak{£}^{*}$. Bearing in mind (2), we have

$$
£^{*}=\phi_{2}\left(X^{*}\right)=\phi_{2}\left(X_{*}\right)=\frac{\phi_{1}\left(X_{*}\right)}{1-\phi_{1}\left(X_{*}\right)}=\frac{£_{*}}{1-£_{*}}
$$

and the remaining properties of Assertion (a) are obvious if one takes into account that $t \leq t /(1-t)$ for every $t \in[0,1)$.

In order to prove the remaining statements, consider $\gamma X_{*}$ with $\gamma>0$ and such that $\gamma P X_{*}^{+}=1$. Since $\phi_{2}$ is homogeneous and $X_{*}$ solves (8), so does $\gamma X_{*}$, i.e., $\phi_{2}$ attains the optimal value at $\gamma X_{*}$. Thus,

$$
\phi_{2}\left(X^{*}\right)=£^{*}=\phi_{2}\left(\gamma X_{*}\right)=\left(-\gamma P X_{*}\right) /\left(\gamma P X_{*}^{+}\right)=-\gamma P X_{*} .
$$

On the other hand, $\gamma P X_{*}^{+}=1$ implies

$$
1=\gamma P X_{*}^{+}=\gamma\left(P X_{*}+P X_{*}^{-}\right)=\gamma\left(-\mathfrak{£}_{*}+1\right)
$$

and

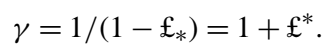


Let us introduce the TSIR envelopes $\mu_{*}$ and $\mu^{*}$ and the theoretical prices $P_{*}=$ $\mu_{*} A$ and $P^{*}=\mu^{*} A,\left(\mathfrak{f}_{*}, \lambda_{*}, \mu_{*}\right)$ and $\left(\mathfrak{£}^{*}, \lambda^{*}, \mu^{*}\right)$ being solutions of (4) and (5). According to Theorem 3.1 both price vectors generate SSA-free markets.

\section{Theorem 3.3}

(a) $p_{* j} \leq p_{j} \leq p_{j}^{*}, j=1,2, \ldots, n$.

(b) If $k_{j}^{*}>0$, then $p_{* j}=p_{j}=p_{j}^{*} \frac{1}{1+£^{*}}$. If $h_{* j}>0$, then $p_{* j} \frac{1}{1-£_{*}}=p_{j}=p_{j}^{*}$.

(c) $P_{*} X \leq P X \leq P^{*} X$ for every $X \geq 0$. Furthermore, the first (second) expression holds in terms of equality if $X$ is composed of those bonds of $k^{*}\left(h_{*}\right)$.

Proof (a) is obvious because $\lambda_{*}, \lambda^{*}, A \geq 0$, and (c) trivially follows from (a) and (b). Besides, both assertions in (b) are analogous, so let us prove the first one. Assume that $k_{j}^{*}>0$. Then Lemma 3.2 ensures that $x_{j}^{*}>0$. Consequently, Theorem $3.2 \mathrm{~b}$ shows that $x_{* j}>0$. The complementary slackness conditions between (2) and (4) show that $\lambda_{* j}=0$, and therefore, $p_{* j}=p_{j}$. Besides, the complementary slackness conditions between (3) and (5) show that $\left(£^{*} P-\lambda^{*}\right) k^{*}=0$, and thus $\lambda^{*} k^{*}=£^{*} P k^{*}=£^{*}$. Hence,

$$
P^{*} k^{*}=\mu^{*} A k^{*}=\left(P+\lambda^{*}\right) k^{*}=1+£^{*} .
$$

Furthermore, the constraints (5) guarantee that $\lambda_{j}^{*} \leq £^{*} p_{j}$. Thus,

$$
p_{j}^{*}-p_{j} \leq £^{*} p_{j},
$$

and therefore,

$$
p_{j}^{*} \leq p_{j}\left(1+£^{*}\right)
$$

for $j=1,2, \ldots, n$. Suppose that

$$
p_{j_{0}}^{*}<p_{j_{0}}\left(1+£^{*}\right)
$$

holds for some $j_{0}$ with $k_{j_{0}}^{*}>0$. Then, (10) leads to

$$
P^{*} k^{*}<\left(1+£^{*}\right) P k^{*}=1+£^{*},
$$

contradicting (9).

Remark 3.1 Lemma 3.1 and Theorem 3.2 point out that $£_{*}$ and $£^{*}$ are adequate measures of the level of SSA, since the arbitrage earnings grow as do the measures. Furthermore, since $[0,1) \ni t \longrightarrow t /(1-t) \in[0, \infty)$ is a one to one increasing function, both measures yield similar information about the degree of SSA, and lead to similar optimal arbitrage strategies due to Theorem 3.2(b). Besides, Theorem 3.3 shows that both TSIR envelopes $\mu_{*}$ and $\mu^{*}$ must be considered when providing upper and lower bounds for real market prices. The use of envelopes seems to be more accurate than the use of a single TSIR since the computation of bounds is far more precise than the computation of approximations for prices without information about the committed errors. Finally, notice that Theorem 3.3(b) emphasizes the relationships between both 
SSA arbitrage measures. Indeed, the solution of (5) (dual problem of (3)) matches the prices of the bonds included in the second component of the solution of (2) and vice versa.

Another interesting property of $£_{*}$ and $\mathfrak{f}^{*}$ is that they minimize the maximum variation (in percentage) of prices preventing the existence of SSA.

Proposition 3.1 Let $Q=\left(q_{1}, q_{2}, \ldots, q_{n}\right)$ be a vector of prices for $B_{1}, B_{2}, \ldots, B_{n}$ that generates a SSA-free market and such that $0<q_{j} \leq p_{j}, j=1,2, \ldots, n$. Then,

$$
f_{*}=\operatorname{Max}\left\{\frac{p_{j}-p_{* j}}{p_{j}}: j=1,2, \ldots, n\right\} \leq \operatorname{Max}\left\{\frac{p_{j}-q_{j}}{p_{j}}: j=1,2, \ldots, n\right\} .{ }^{2}
$$

Proof Assume that $\mathfrak{f}_{*}>0$, since the result is obvious otherwise. The constraints (5) lead to $\mathfrak{f}_{*} \geq \frac{\lambda_{* j}}{p_{j}}=\frac{p_{j}-p_{* j}}{p_{j}}, j=1,2, \ldots, n$ and Theorem 3.3(b) shows that $\mathfrak{f}_{*}=$ $\frac{p_{j}-p_{* j}}{p_{j}}$ if $h_{* j}>0$. On the other hand, since $Q$ leads to a SSA-free market, Theorem 3.1 guarantees the existence of $\mu=\left(\mu_{1}, \mu_{2}, \ldots, \mu_{m}\right)$ such that $\mu A=Q$ and $\mu_{1} \geq \mu_{2} \geq \cdots \geq \mu_{m} \geq 0$. Consider

$$
\lambda=P-\mu A=P-Q \geq 0
$$

and

$$
\theta=\operatorname{Max}\left\{\frac{p_{j}-q_{j}}{p_{j}}: j=1,2, \ldots, n\right\} .
$$

Then, $(\mu, \lambda, \theta)$ is (5)-feasible and therefore $\theta \geq \mathfrak{f}_{*}$.

Remark 3.2 The measurement of the degree of STSA is quite similar, though there are some minor differences. Consider the problems

$$
\begin{aligned}
& \operatorname{Max}-P X, \\
& \text { s.t. } I_{m}^{* *} \tilde{A} X \geq 0, \\
& \quad x_{j}+h_{j} \geq 0, \quad j=1,2, \ldots, n, \\
& \quad \sum_{j=1}^{n} h_{j} p_{j} \leq 1, \\
& \quad h_{j} \geq 0, \quad j=1,2, \ldots, n,
\end{aligned}
$$

and

$$
\begin{aligned}
& \operatorname{Max}-P X, \\
& \text { s.t. } I_{m}^{* *} \tilde{A} X \geq 0,
\end{aligned}
$$

\footnotetext{
${ }^{2}$ An analogous result holds for $£^{*}, P^{*}$ and $Q \geq P$
} 


$$
\begin{aligned}
& x_{j}-k_{j} \leq 0, \quad j=1,2, \ldots, n, \\
& \sum_{j=1}^{n} k_{j} p_{j} \leq 1, \\
& k_{j} \geq 0, \quad j=1,2, \ldots, n,
\end{aligned}
$$

$(X, h) \in \mathbb{R}^{n} \times \mathbb{R}^{n}$ and $(X, k) \in \mathbb{R}^{n} \times \mathbb{R}^{n}$ being the decision variables. The economic interpretation is similar to that discussed when dealing with SSA. Their duals are

$$
\begin{aligned}
& \text { Min } \theta, \\
& \text { s.t. } \mu A \pm \lambda=P\left(1+\mu_{1}\right), \\
& \quad \lambda_{j} \leq \theta p_{j}, \quad j=1,2, \ldots, n, \\
& \quad \mu_{1} \geq \mu_{2} \geq \cdots \geq \mu_{m} \geq 0, \\
& \quad \lambda_{j} \geq 0, \quad j=1,2, \ldots, n,
\end{aligned}
$$

where the symbol $+(-)$ in the first constraint is used for the dual of (11), (12). Denote by $£_{* *}$ and $£^{* *}$ the optimal values. Due to the presence of $\mu_{1}$ on the right-hand side of (13), things are not absolutely similar when we look for those modification of prices leading to STSA-free markets. So, take $\mu_{* *}, \mu^{* *} \in \mathbb{R}^{m}$ solving (13), and set the TSIR envelopes and theoretical prices

$$
\begin{aligned}
& \tilde{\mu}_{* *}=\left(\mu_{* *}\right) /\left(1+\mu_{* * 1}\right), \quad \tilde{\mu}^{* *}=\left(\mu^{* *}\right) /\left(1+\mu_{1}^{* *}\right), \\
& \tilde{P}_{* *}=\tilde{\mu}_{* *} A \text { and } \tilde{P}^{* *}=\tilde{\mu}^{* *} A .
\end{aligned}
$$

Define the STSA measures as

$$
\tilde{\mathfrak{E}}_{* *}=\mathfrak{£}_{* *} /\left(1+\mu_{* * 1}\right) \quad \text { and } \quad \tilde{\mathfrak{£}}^{* *}=\mathfrak{£}^{* *} /\left(1+\mu_{1}^{* *}\right) .
$$

Proceeding as in Theorems 3.3, the absence of STSA may be characterized by the existence of $\tilde{\mu}_{* *}$ such that

$$
P=\tilde{\mu}_{* *} A \quad \text { and } \quad 1>\tilde{\mu}_{* * 1} \geq \tilde{\mu}_{* * 2} \geq \cdots \geq \tilde{\mu}_{* * m} \geq 0,
$$

and Theorems 3.2 and 3.3 may also be extended in a straightforward manner.

Let us compare both approaches. If we analyze the presence of STSA and use it to estimate the TSIR or its envelopes there is and advantage since we will never obtain discount factors larger than one. However, $£_{*}$ and $£^{*}$ are relative arbitrage gains and therefore these measures more appropriately reflect the level of arbitrage. If they are large then the arbitrage profits are large, and prices must be modified according to their large values in order to prevent the existence of SSA. On the contrary, $\mathfrak{£}_{* *}$ and $£^{* *}$ reflect discrepancies in prices of similar portfolios and, therefore, they are relative arbitrage incomes, but not profits (initial incomes may be used to overcome possible negative cash flows). Accordingly, they have to be modified according to (14) in order to provide us with the variation of prices generating a STSA-free market. They may be large even if $\tilde{\mathfrak{E}}_{* *}$ and $\tilde{\tilde{f}}^{* *}$ achieve small values and slight modifications of prices eliminate the presence of STSA. 


\section{Further Applications: Embedded Options and Credit Risk Spreads}

The methodology above enables us to estimate the TSIR by also incorporating the information contained in extendible or callable bonds. The empirical evidence shows that it is not convenient to eliminate these bonds because the TSIR generated by option-free assets may be not very accurate and lead to negative embedded options prices. This fact has been pointed out by [10] for the U.S. market and [11] for the Canadian market, amongst others. This caveat has been addressed in [12] by applying what the author calls "an implied norm approach". We propose here an alternative procedure.

Henceforth, we will impose the absence of arbitrage, and notice that this assumption does not imply that the measures above vanish if extendible or callable bonds are involved. Indeed, positive values of the measures could lead to apparent arbitrage portfolios that give up being arbitrage if one considers the effect of the embedded option.

Proposition 4.1 Suppose that $B_{1}, B_{2}, \ldots, B_{n-k}$ are option-free and $B_{n-k+1}, \ldots, B_{n}$ are extendible (callable). Let $\left\{q_{n-j} ; j=0, \ldots, k-1\right\}$ be the prices of the optionfree bonds with the same payoffs as $B_{n-j}$. Let $\left\{v_{n-j} ; j=0, \ldots, k-1\right\}\left(\left\{c_{n-j} ; j=\right.\right.$ $0, \ldots, k-1\})$ be the prices of the embedded puts (calls). Let $r$ be such that

$$
\begin{aligned}
\frac{v_{r}}{p_{r}} & =\operatorname{Max}\left\{\frac{v_{n-j}}{p_{n-j}} ; j=0, \ldots, k-1\right\}, \\
\left(\frac{c_{r}}{p_{r}}\right. & \left.=\operatorname{Max}\left\{\frac{c_{n-j}}{p_{n-j}} ; j=0, \ldots, k-1\right\}\right) .
\end{aligned}
$$

Then

$$
\begin{aligned}
& q_{r} \leq p_{r}\left(1-£_{*}\right) \quad \text { and } \quad v_{r} \geq p_{r} £_{*}, \\
& \left(q_{r} \geq p_{r}\left(1+£^{*}\right) \quad \text { and } \quad c_{r} \leq p_{r} £^{*}\right) .
\end{aligned}
$$

Proof We will only prove the result for extendible bonds. The equality $p_{n-j}=$ $q_{n-j}+v_{n-j}$ obviously holds. Since $\left\{p_{1}, \ldots, p_{n-k}, q_{n-k+1}, \ldots, q_{n}\right\}$ is a set of prices preventing the existence of SSA, Proposition 3.1 shows that

$$
\frac{p_{r}-q_{r}}{p_{r}}=\operatorname{Max}\left\{\frac{p_{n-j}-q_{n-j}}{p_{n-j}} ; j=0, \ldots, k\right\} \geq \mathfrak{£}_{*},
$$

and the conclusion trivially follows.

Remark 4.1 The latter result provides us with a method to solve the "negative embedded option price puzzle". Indeed, suppose for instance that we are dealing with extendible securities. For $r=n-k+1, \ldots, n$, we can compute $\mathfrak{f}_{*}^{r}$, the value of $\mathfrak{f}_{*}$ for the set of bonds $\left\{B_{1}, B_{2}, \ldots, B_{n-k}, B_{r}\right\}$. Then, we have the lower bounds

$$
v_{r} \geq p_{r} \mathfrak{£}_{*}^{r},
$$


and we look for vectors $\mu$ satisfying

$$
\begin{aligned}
& \mu_{1} \geq \cdots \geq \mu_{m} \geq 0, \\
& \mu C_{j}=p_{j}, \quad j=1, \ldots, n-k,
\end{aligned}
$$

and

$$
\mu C_{j} \leq p_{j}\left(1-\mathfrak{f}_{*}^{j}\right), \quad j=n-k+1, \ldots, n .
$$

The selection of $\mu$ may be implemented by optimization methods. For instance, "Generalized Least Squares" with respect to that $\mu$ ' that we would obtain if we removed the extendible assets.

The method above may be improved if we are able to rank the options according to the ratio in (15). Indeed, in such a case, we can calculate each $£_{*}^{r}$ by considering sets of bonds containing more than one extendible bond, which makes $£_{*}^{r}$ and the lower bond $p_{r} £_{*}^{r}$ increase.

An alternative method consists of solving problem (4) with the second constraint relaxed to $\lambda_{j} \leq \theta p_{j}, j=n-k+1, \ldots, n$. Denoting by $\theta_{*}$ the optimal value, we can modify the prices of those extendible bonds saturating this constraint according to $q_{r} \leq p_{r}\left(1-\theta_{*}\right)$. Remove the bonds whose prices have been changed and repeat the process several times if necessary, so as to ensure that all the extendible bonds have been removed. Then, compute $\mu$ as said above.

Finally, if $B_{1}, B_{2}, \ldots, B_{n-k}$ are option-free and default-free and if $B_{n-k+1}, \ldots, B_{n}$ are option-free and nondefault-free private bonds, then Proposition 4.1 and the procedure above may be readapted in a straightforward way so as to yield lower bounds for the credit risk spread of $B_{n-k+1}, \ldots, B_{n}$. Moreover, one can compute TSIR proxies that incorporate the information contained in nondefault-free securities.

\section{Empirical Test}

The existence of sequential arbitrage has been tested in the Spanish market. We used the database of daily prices provided by the Bank of Spain that contains the price of real transactions corresponding to default-free bonds issued by the Spanish government, bonds issued by the regional governments of several Spanish communities and bonds with embedded options also issued by regional governments. We have focused on the period between years 1994 and 2002, though the empirical results have been quite homogeneous regardless of the tested year.

Regarding the existence of sequential arbitrage portfolios composed of defaultfree and option-free bonds, Table 1 summarizes some results of 2002. It is clear that the sequential arbitrage existence cannot be rejected. The existence of SSA-arbitrage and STSA-arbitrage are closely related, and the values of $\mathfrak{£}_{*}$ and $\tilde{\mathfrak{f}}_{* *}$ are almost equal. With respect to the TSIR estimation, the usual shape of $\mu_{*}$ and $\mu^{*}$ is provided in Fig. 1.

The average value of the credit risk spread of those bonds issued by the regional governments is low and close to $0.08 \%$ of the total bond price, which implies that investors have a high degree of confidence in these assets. The last test also incorporates extendible and-or callable bonds issued by local governments. Table 1 
Table 1

\begin{tabular}{|c|c|c|c|c|c|c|}
\hline \multirow[b]{2}{*}{ Value of $\tilde{£}_{* *}, \mathfrak{£}_{*}$} & \multicolumn{2}{|c|}{$\begin{array}{l}\text { Default-free and } \\
\text { option-free bonds } \\
\% \text { of days with } \\
\tilde{£}_{* *}, £_{*}>0\end{array}$} & \multicolumn{2}{|c|}{$\begin{array}{l}\text { Option-free bonds } \\
\text { with risk premium } \\
\% \text { of days with } \\
\tilde{\mathfrak{E}}_{* *}, \mathfrak{f}_{*}>0 \\
\end{array}$} & \multicolumn{2}{|c|}{$\begin{array}{l}\text { Risk premium and } \\
\text { embedded option } \\
\% \text { of days with } \\
\tilde{\mathfrak{f}}_{* *}, \mathfrak{f}_{*}>0 \\
\end{array}$} \\
\hline & STSA & SSA & STSA & SSA & STSA & SSA \\
\hline$£_{* *}<0.001$ & 64.20 & 64.63 & 59.92 & 60.32 & 58.70 & 59.11 \\
\hline $0.001 \leq \tilde{\mathfrak{f}}_{* *}, \mathfrak{f}_{*}<0.0025$ & 22.80 & 22.36 & 21.86 & 21.46 & 21.46 & 21.05 \\
\hline $0.0025 \leq \tilde{\mathfrak{f}}_{* *}, \mathfrak{f}_{*}<0.005$ & 8.13 & 8.13 & 10.53 & 10.53 & 10.53 & 10.53 \\
\hline $0.005 \leq \tilde{\mathfrak{f}}_{* *}, \mathfrak{f}_{*}<0.01$ & 3.66 & 3.66 & 6.57 & 6.07 & 6.07 & 6.48 \\
\hline $0.01 \leq \tilde{\mathfrak{E}}_{* *}, \mathfrak{£}_{*}<0.02$ & 0.81 & 0.81 & 1.62 & 1.21 & 1.62 & 1.21 \\
\hline $0.02 \leq \tilde{\mathfrak{E}}_{* *}, \mathfrak{f}_{*}<0.05$ & 0.41 & 0.41 & 0.40 & 0.40 & 0.40 & 0.40 \\
\hline $0.05 \leq \tilde{\mathfrak{f}}_{* *}, \mathfrak{f}_{*}<2.5$ & 0 & 0 & 0 & 0 & 1.21 & 1.21 \\
\hline
\end{tabular}

Fig. 1 December 14th, 1998 The market reflects sequential arbitrage opportunities and $\tilde{£}_{* *}=0.003554708$ The continuous lines represent the estimation of $\mu_{*}$ and $\mu^{*}$

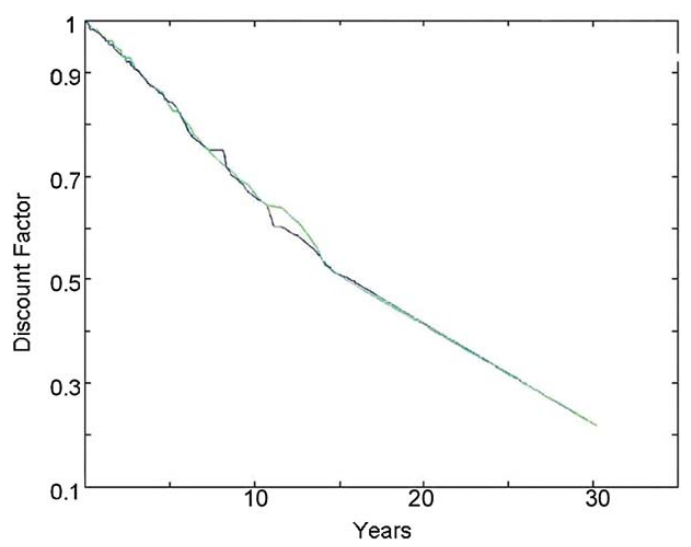

summarizes the results in 2002. Measures $\mathfrak{f}_{*}$ and $\tilde{\mathfrak{f}}_{* *}$ are almost equal once more. For those days when the option-free bonds market was arbitrage-free we have obtained the embedded option premium, whose average value is $10 \%$ of the total bond price.

Summarizing, our results seem to reveal some inefficiencies in the market and, therefore, the existence of sequential arbitrage cannot be rejected. The credit risk associated with the local governments of the Spanish communities is rather small, and the embedded option average price is close to $10 \%$. The set of TSIR envelopes always matches the price of the available bonds. 


\section{Conclusions}

This paper has introduced new measures that allow us to study the existence of sequential arbitrage in bond markets. The measures are defined by means of two dual pairs of optimization problems.

Primal problems provide concrete sequential arbitrage portfolios (if they exist) and show that the value of the measures may be interpreted in monetary terms. Thus, the effect of imperfections (broker's commissions, for example) may be discounted in practical applications.

Dual problems provide envelopes for the TSIR and show that the value of the measures may be interpreted in terms of "pricing errors". They indicate how real prices must be modified (in percentages) to prevent the existence of sequential arbitrage.

Every dual pair of optimization problems generates two envelopes for the TSIR. The envelopes allow us to establish upper and lower bounds for the price of every portfolio. This is a clear advantage with respect to the usual approach, which considers a single TSIR that only approximates securities prices without information about the committed errors.

The TSIR is usually estimated by only drawing on default free and option free bonds. This is a clear limitation that has led to the negative embedded option price puzzle. The introduced methodology may give a practical solution to this problem.

\section{References}

1 Luenberger, D G : Projection pricing J Optim Theory Appl 109, 1-25 (2001)

2 Luenberger, D G : Pricing a nontradeable asset and its derivatives J Optim Theory Appl 121(3), 465-487 (2004)

3 Hansen, L , Jagannathan, R : Assessing specification errors in stochastic discount factor models J Finance 52(2), 567-590 (1997)

4 Chen, Z, Knez, PJ : Measurement of market integration and arbitrage Rev Financ Stud 8(2), 545560 (1995)

5 Kempf, A, Korn, O : Trading system and market integration J Financ Intermed 7, 220-239 (1998)

6 Balbás, A, Guerra, P J , Muñoz-Bouzo, M J : The balance space approach with Riesz spaces valued objectives: An application to financial markets Comput Math Appl 44(7), 887-897 (2002)

7 Jaschke, S R : Arbitrage bounds for the term structure of interest rates Finance Stoch 2, 29-40 (1998)

8 Nakano, Y: Efficient hedging with coherent risk measure J Math Anal Appl 293, 345-354 (2004)

9 Rockafellar, R T, Uryasev, S, Zabarankin, M : Optimality conditions in portfolio analysis with general deviations measures Math Program B 108, 515-540 (2006)

10 Longstaff, FA : Are negative option prices possible? The callable U S treasury-bond puzzle J Bus 65(4), 571-592 (1992)

11 Athanassakos, G, Carayannopoulos, P, Tian, Y: Negative option values in extendible Canadian treasury bonds Adv Futures Options Res 9, 83-110 (1994)

12 Ioffe, I D : Arbitrage bounds in markets with noisy prices and the puzzle of negative option prices implicit in bonds J Bank Finance 26(6), 1199-1228 (2002) 